\title{
A scalable, line-independent control design algorithm for voltage and frequency stabilization in AC islanded microgrids
}

\author{
Michele Tucci $^{\mathrm{a}}$, Giancarlo Ferrari-Trecate ${ }^{\mathrm{b}}$ \\ ${ }^{a}$ Dipartimento di Ingegneria Industriale e dell'Informazione, Università degli Studi di Pavia, Italy \\ b Automatic Control Laboratory, École Polytechnique Fédérale de Lausanne (EPFL), Switzerland
}

\begin{abstract}
We propose a decentralized control synthesis procedure for stabilizing voltage and frequency in AC Islanded microGrids (ImGs) composed of Distributed Generation Units (DGUs) and loads interconnected through power lines. The presented approach enables Plug-and-Play (PnP) operations, meaning that DGUs can be added or removed without compromising the overall ImG stability. The main feature of our design algorithm is that it does not depend on line parameters. This implies that (i) the synthesis of each local controller requires only the model of the corresponding DGU, and (ii) whenever a new DGU is plugged in, neighboring DGUs do not have to retune their regulators because of the new power line connected to them. Moreover, we formally prove that stabilizing local controllers can be always computed, independently of the electrical parameters. Theoretical results are validated by simulating in PSCAD the behavior of a 10-DGUs ImG.
\end{abstract}

Key words: Decentralized control, voltage control, asymptotic stability, microgrid, renewable energy systems.

\section{Introduction}

Voltage and frequency stability is a central problem in low-voltage AC Islanded microGrids (ImGs) that has received great attention within the control and the power electronics communities (Guerrero et al. 2013). In absence of a connection to the main grid (which acts as an infinite power source and as a master clock for the ImG frequency), voltage and frequency must be governed by the local controllers of the Voltage Source Converters (VSCs) interfacing power sources with the ImG. Each controlled VSC, together with its power supply, forms a Distributed Generation Unit (DGU) connected to loads and other DGUs through power lines. Voltage and frequency control can be then formulated as the problem of designing decentralized regulators guaranteeing collective ImG stability in spite of the electrical coupling between DGUs. ImGs are usually equipped with hierarchical controllers and voltage regulators are embed-

\footnotetext{
^ This work has received support from the Swiss National Science Foundation under the COFLEX project (grant number 200021_169906). Michele Tucci is currently with Bombardier Transportation, Zürich, Switzerland.

Email addresses:

michele.tucci02@universitadipavia.it (Michele Tucci), giancarlo.ferraritrecate@epfl.ch (Giancarlo Ferrari-Trecate).
}

ded into the primary layer (Guerrero et al. 2013). Approaches to the decentralized control of ImGs can be divided into two main classes. The first one embraces droop controllers (Guerrero et al. 2013), which admit a simple implementation and do not require synchronized DGU clocks for the computation of the control variables. However, the droop method can generate frequency deviations, whose compensation calls for the use of secondary controllers (Guerrero et al. 2013) or communication networks (Kolluri et al. 2018). Stability properties of droopcontrolled microgrids have been analyzed in (Schiffer et al. 2014, Simpson-Porco et al. 2013) under simplified models of the DGU dynamics. The second class of controllers comprises droop-free regulators often based on approaches developed within the field of decentralized control (Etemadi et al. 2012, Babazadeh and Karimi 2013, Riverso et al. 2015). These methods usually require controller clocks to be synchronized with sufficient precision. To this purpose, technologies based on communication networks are available. For instance, the precision Time Protocol IEEE standard (IEEE1588 2008, IEEE1588 2017) describes architectures based on GPS and Ethernet for providing clock accuracy of $100 \mathrm{~ns}-1 \mu \mathrm{s}$ by utilizing rather infrequent updates (every $10 \mathrm{~ms}-1 \mathrm{~s}$ ). Other synchronization methods for ImGs are discussed in (Etemadi et al. 2012, Riverso et al. 2018) and their applicability has been shown in lab experiments. 
The necessity to address the growing demand for flexible and resizable ImGs, where DGUs and loads can enter/leave over time, calls for control architectures that can be easily updated when the ImG topology changes. Approaches of this kind have been often termed Plugand-Play (PnP) (Sadabadi et al. 2017, Tucci et al. 2018, Riverso et al. 2015, Dörfler et al. 2014). In particular, for the PnP design algorithm in (Riverso et al. 2015) (i) the computation of a local controller for a DGU amounts to an optimization problem based only the DGU model and the parameters of power lines connected to it, (ii) stability of the ImG can be guaranteed. Note that, from (i), the plug-in of a DGU requires to update controllers of neighboring DGUs and it is denied if controllers for the DGU and its neighbors cannot be computed.

In this paper, we develop a PnP control design method that, similarly to (Riverso et al. 2015), assumes synchronization of the DGU clocks. However, differently from (Riverso et al. 2015), it does not require knowledge of power lines, whose parameters are often uncertain; the only global quantity used in the synthesis algorithm is a scalar parameter. We do not assume either to know bounds on electrical coupling parameters, as done in (Sadabadi et al. 2017). These simplifications are desirable for several reasons. First, the addition/removal of a DGU does not require to update any existing controller in the ImG. Indeed, plugging in/out operations do add/remove lines connected to neighboring DGUs, but DGU controllers are line-independent. Second, while the PnP design in (Riverso et al. 2015) depends on a tuning parameter, which must be sufficiently small for ensuring collective ImG stability, here this constraint is removed. To this purpose, as in (Riverso et al. 2015), we assume Quasi Stationary Line (QSL) approximations. However, stability is shown using a novel argument based on the Laplacian structure of the admittance matrix representing DGU interconnections. Finally, differently from (Riverso et al. 2015), we show that local regulators always exist, and they can be computed by solving Linear Matrix Inequality (LMI) problems. Hence, the plugin/out of a DGU is never denied.

The approach taken in this paper shares similarities with the one in (Tucci et al. 2018), where DC mGs are considered. There are, however, fundamental differences. First, in the AC case, one must handle three-phase balanced signals or, in an equivalent way, their $d q$ representation (Schiffer et al. 2016). This makes stability analysis more complex and, differently from (Tucci et al. 2018), our rationale hinges on a suitable reparametrization of local controllers and Lyapunov functions. Second, compared to (Tucci et al. 2018) the LMIs associated to local control design involve a different set of optimization variables and are guaranteed to be feasible.

The paper is structured as follows. After introducting the ImG model and the local control architecture (Section 2), we describe the line-independent, LMI-based, $\mathrm{PnP}$ design procedure in Section 3. In Section 4, we discuss simulation results using a 10-DGU ImG and comment on the impact of clock desynchronization on stability. A preliminary version of this work has been presented in the conference paper (Tucci and FerrariTrecate 2017).

Notation and basic definitions. The identity and null matrices of size $N$ are denoted, respectively, with $I_{N}$ and $\mathbf{0}_{N}$, while we use $\mathbf{0}$ to indicate a null matrix of appropriate size. The inequality $A>0$ means that the matrix $A \in \mathbb{R}^{n \times n}$ is positive definite.

\section{Microgrid model}

In this Section, we briefly introduce the electrical model of the ImG, deferring the reader to (Riverso et al. 2015, Floriduz et al. 2018) for details. We assume three-phase electrical signals without zero-sequence components and balanced network parameters. The single-phase equivalent scheme of DGU $i$, shown in the left dashed frame of Figure 1, includes: a DC voltage source (modeling a generic renewable), a controlled VSC and a local load, connected to the Point of Common Coupling (PCC) through an $R L C$ filter ${ }^{1}$. with parameters $R_{t i}, L_{t i}$ and $C_{t i}$. We further assume that loads $I_{L i}$ are unknown and act as current disturbances (Babazadeh and Karimi 2013). The ImG is composed of $N$ DGUs indexed by the set $\mathcal{D}=\{1, \ldots, N\}$. Then, we call two DGUs neighbors if there is a power line connecting their PCCs and denote with $\mathcal{N}_{i} \subset \mathcal{D}$ the subset of neighbors of DGU $i$. We indicate with $\mathcal{G}_{e l}$ the undirected electric graph induced by the neighboring relation over the node set $\mathcal{D}$. As shown in the right dashed frame of Figure $1, R_{i j}$ and $L_{i j}$ are, respectively, the resistance and inductance of the power line connecting DGUs $i$ and $j$.

Let $\omega_{0}$ be the reference network frequency. Next, we provide a model of DGU $i$ where all electric variables are represented in the $d q$ reference frame rotating with speed $\omega_{0}$. Using the notation of Figure 1, The state of DGU $i$ is $x_{[i]}=\left[V_{i}^{d}, V_{i}^{q}, I_{t i}^{d}, I_{t i}^{q}\right]^{T}$ and collects the $d$ and $q$ components of the PCC voltage and the filter current. The control input is $u_{[i]}=\left[V_{t i}^{d}, V_{t i}^{q}\right]^{T}$ and the load current, which acts as an exogenous disturbance, is $d_{[i]}=\left[I_{L i}^{d}, I_{L i}^{q}\right]^{T}$. The measured output and controlled variables are, respectively, $y_{[i]}=x_{[i]}$ and $z_{[i]}=\left[V_{i}^{d}, V_{i}^{q}\right]^{T}$.

By using QSL approximations of power lines (Riverso et al. 2015) one obtains the LTI system

$$
\Sigma_{[i]}^{D G U}:\left\{\begin{array}{l}
\dot{x}_{[i]}(t)=A_{i i} x_{[i]}(t)+B_{i} u_{[i]}(t)+M_{i} d_{[i]}(t)+\xi_{[i]}(t) \\
y_{[i]}(t)=C_{i} x_{[i]}(t) \\
z_{[i]}(t)=H_{i} y_{[i]}(t)
\end{array}\right.
$$

\footnotetext{
1 As shown in (Dörfler and Bullo 2013, Floriduz et al. 2018), also more general interconnections of loads and DGUs can be mapped into this setting by means of Kron reduction.
} 


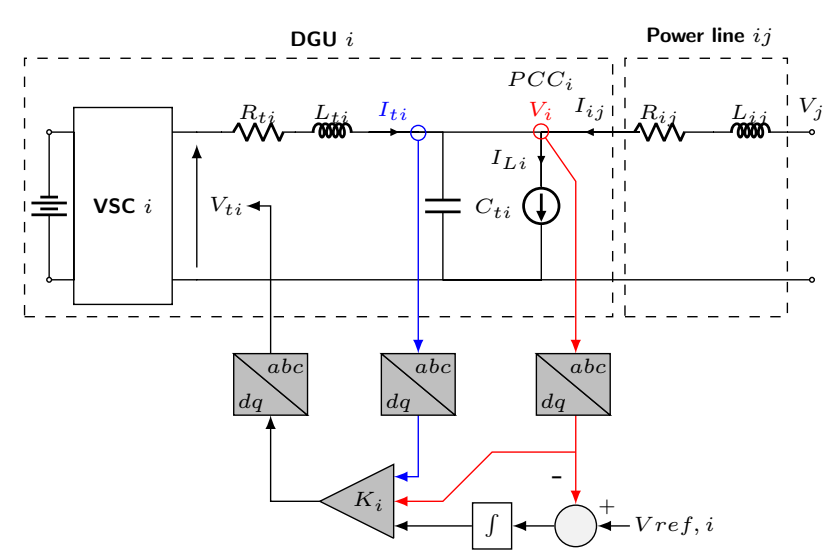

Fig. 1. Electrical scheme of DGU $i$, power line $i j$, and local $\mathrm{PnP}$ voltage and frequency controller.

where $\xi_{[i]}=\sum_{j \in \mathcal{N}_{i}} A_{i j}\left(x_{[j]}-x_{[i]}\right)$ accounts for the coupling with neighboring DGUs through PCC voltages and

$A_{i i}=\left[\begin{array}{cccc}0 & \omega_{0} & \frac{1}{C_{t i}} & 0 \\ -\omega_{0} & 0 & 0 & \frac{1}{C_{t i}} \\ -\frac{1}{L_{t i}} & 0 & -\frac{R_{t i}}{L_{t i}} & \omega_{0} \\ 0 & -\frac{1}{L_{t i}} & -\omega_{0} & -\frac{R_{t i}}{L_{t i}}\end{array}\right], A_{i j}=\frac{1}{C_{t i}}\left[\begin{array}{ccc}\frac{R_{i j}}{Z_{i j}^{2}} & \frac{X_{i j}}{Z_{i j}^{2}} & \mathbf{0} \\ -\frac{X_{i j}}{Z_{i j}^{2}} & \frac{R_{j i}}{Z_{i j}^{2}} & \mathbf{0} \\ \mathbf{0} & \mathbf{0} & \mathbf{0}\end{array}\right]$,

$B_{i}=\left[\begin{array}{cc}\mathbf{0} & \mathbf{0} \\ \frac{1}{L_{t i}} & 0 \\ 0 & \frac{1}{L_{t i}}\end{array}\right], M_{i}=\left[\begin{array}{cc}-\frac{1}{C_{t i}} & 0 \\ 0 & -\frac{1}{C_{t i}} \\ \mathbf{0} & \mathbf{0}\end{array}\right], C_{i}=I_{4}, H_{i}=\left[\begin{array}{lll}1 & 0 & \mathbf{0} \\ 0 & 1 & 0\end{array}\right]$,

where $X_{i j}=\omega_{0} L_{i j}$ and $Z_{i j}=\left|R_{i j}+\mathrm{i} X_{i j}\right|$.

The overall ImG model is given by

$$
\left\{\begin{array}{l}
\dot{\mathbf{x}}(t)=\mathbf{A} \mathbf{x}(t)+\mathbf{B u}(t)+\mathbf{M} \mathbf{d}(t) \\
\mathbf{y}(t)=\mathbf{C x}(t) \\
\mathbf{z}(t)=\mathbf{H y}(t)
\end{array}\right.
$$

where $\mathbf{x}=\left[x_{[1]}^{T}, \ldots, x_{[N]}^{T}\right]^{T} \in \mathbb{R}^{4 N}$, and vectors $\mathbf{u}, \mathbf{d}, \mathbf{y}$, and $\mathbf{z}$ are similarly defined. Matrices $\mathbf{A}, \mathbf{B}, \mathbf{M}, \mathbf{C}$ and $\mathbf{H}$, which can be easily derived from (2)-(3), are provided in Appendix A.3 of (Riverso et al. 2014).

\section{Design of stabilizing controllers}

As in (Riverso et al. 2015), the goal of local controllers is to guarantee offset-free tracking of constant references $\mathbf{z}_{\mathbf{r e f}}(t)=\overline{\mathbf{z}}_{\mathbf{r e f}}$. Note that, as show in Figure 1, local references $z_{r e f_{[i]}}=V_{\text {ref, } i}$ are $d q$ signals and therefore provide complete information about the desired amplitude and frequency for the voltage $V_{i}$. Typically, $z_{r e f_{[i]}}$ is provided by higher control layers devoted to power flow regulation (Guerrero et al. 2013, Riverso et al. 2018). For offset-free tracking, the ImG model is augmented with the integrators

$$
\begin{aligned}
\dot{v}_{[i]}(t)=e_{[i]}(t) & =z_{r e f[i]}(t)-z_{[i]}(t) \\
& =z_{r e f[i]}(t)-H_{[i]} C_{[i]} x_{[i]}(t),
\end{aligned}
$$

also shown in Figure 1. The augmented DGU model is

$$
\hat{\Sigma}_{[i]}^{D G U}:\left\{\begin{array}{l}
\dot{\hat{x}}_{[i]}(t)=\hat{A}_{i i} \hat{x}_{[i]}(t)+\hat{B}_{i} u_{[i]}(t)+\hat{M}_{i} \hat{d}_{[i]}(t)+\hat{\xi}_{[i]}(t) \\
\hat{y}_{[i]}(t)=\hat{C}_{i} \hat{x}_{[i]}(t) \\
z_{[i]}(t)=\hat{H}_{i} \hat{y}_{[i]}(t)
\end{array}\right.
$$

where $\hat{x}_{[i]}=\left[x^{T}{ }_{[i]}, v_{[i]}^{T}\right]^{T} \in \mathbb{R}^{6}$ is the state, $\hat{y}_{[i]}=\hat{x}_{[i]}$ the measurable output, $\hat{d}_{[i]}=\left[d_{[i]}^{T}, z_{r e f}^{T}{ }_{[i]}^{T}\right]^{T} \in \mathbb{R}^{4}$ the exogenous signals and $\hat{\xi}_{[i]}=\sum_{j \in \mathcal{N}_{i}} \hat{A}_{i j}\left(\hat{x}_{[j]}-\hat{x}_{[i]}\right)$. Moreover, matrices in (5) have the form

$$
\begin{aligned}
\hat{A}_{i i} & =\left[\begin{array}{cc}
A_{i i} & \mathbf{0} \\
\hline-H_{i} C_{i} \mid & \mathbf{0}
\end{array}\right]=\left[\begin{array}{cc|c}
\hat{\mathcal{A}}_{11, i} & \frac{1}{C_{t i}} I_{2} & \mathbf{0}_{2} \\
-\frac{1}{L_{t i}} I_{2} & \hat{\mathcal{A}}_{22, i} & \mathbf{0}_{2} \\
\hline-I_{2} & \mathbf{0}_{2} & \mathbf{0}_{2}
\end{array}\right], \\
\hat{\mathcal{A}}_{11, i} & =\omega_{0}\left[\begin{array}{cc}
0 & 1 \\
-1 & 0
\end{array}\right], \quad \hat{\mathcal{A}}_{22, i}=\omega_{0}\left[\begin{array}{cc}
-\frac{R_{t i}}{L_{t i}} & \omega_{0} \\
-\omega_{0} & -\frac{R_{t i}}{L_{t i}}
\end{array}\right], \\
\hat{A}_{i j} & =\left[\begin{array}{cc}
A_{i j} & \mathbf{0} \\
\mathbf{0} & \mathbf{0}
\end{array}\right], \quad \hat{B}_{i}=\left[\begin{array}{c}
B_{i} \\
\mathbf{0}
\end{array}\right], \\
\hat{C}_{i} & =\left[\begin{array}{cc}
C_{i} & \mathbf{0} \\
\mathbf{0} & I_{2}
\end{array}\right], \quad \hat{M}_{i}=\left[\begin{array}{cc}
M_{i} & \mathbf{0} \\
\mathbf{0} & I_{2}
\end{array}\right], \quad \hat{H}_{i}=\left[\begin{array}{ll}
H_{i} & \mathbf{0}
\end{array}\right] .
\end{aligned}
$$

Since the pair $\left(\hat{A}_{i i}, \hat{B}_{i}\right)$ is controllable (see Proposition 2 in (Riverso et al. 2015)), system (5) can be stabilized in absence of coupling.

As in (Riverso et al. 2015), the overall augmented system is obtained from (5) as

$$
\left\{\begin{array}{l}
\dot{\hat{\mathbf{x}}}(t)=\hat{\mathbf{A}} \hat{\mathbf{x}}(t)+\hat{\mathbf{B}} \mathbf{u}(t)+\hat{\mathbf{M}} \hat{\mathbf{d}}(t) \\
\hat{\mathbf{y}}(t)=\hat{\mathbf{C}} \hat{\mathbf{x}}(t) \\
\mathbf{z}(t)=\hat{\mathbf{H}} \hat{\mathbf{y}}(t)
\end{array}\right.
$$

where $\hat{\mathbf{x}}, \hat{\mathbf{y}}$ and $\hat{\mathbf{d}}$ collect variables $\hat{x}_{[i]}, \hat{y}_{[i]}$ and $\hat{d}_{[i]}$, respectively, and matrices $\hat{\mathbf{A}}, \hat{\mathbf{B}}, \hat{\mathbf{C}}, \hat{\mathbf{M}}$ and $\hat{\mathbf{H}}$ are derived directly from (5).

We equip each DGU $\hat{\Sigma}_{[i]}^{D G U}$ with the following statefeedback controller (see Figure 1),

$$
\mathcal{C}_{[i]}: \quad u_{[i]}(t)=K_{i} \hat{y}_{[i]}(t)=K_{i} \hat{x}_{[i]}(t), \quad K_{i}=\mathbb{R}^{2 \times 6}
$$

inducing a decentralized control architecture. Moreover, if the controllers guarantee closed-loop stability of (6), offset-free tracking is guaranteed as well (Skogestad and Postlethwaite 1996). 


\subsection{Local conditions implying $\operatorname{Im} G$ stability}

If coupling terms $\hat{\xi}_{i}(t)$ are not present, the asymptotic stability of the overall ImG can be ensured by simply stabilizing each closed-loop subsystem

$$
\dot{\hat{x}}_{[i]}(t)=F_{i} \hat{x}_{[i]}(t)+\hat{M}_{i} \hat{d}_{[i]},
$$

where, by construction, thematrix $F_{i}=\hat{A}_{i i}+\hat{B}_{i} K_{i}$ has the following structure

$$
F_{i}=\left[\begin{array}{ccc}
\mathcal{F}_{11, i} & \mathcal{F}_{12, i} & \mathbf{0}_{2} \\
\mathcal{F}_{21, i} & \mathcal{F}_{22, i} & \mathcal{F}_{23, i} \\
-I_{2} & \mathbf{0}_{2} & \mathbf{0}_{2}
\end{array}\right], \quad \mathcal{F}_{11, i}=\hat{\mathcal{A}}_{11, i}, \quad \mathcal{F}_{12, i}=\frac{1}{C_{t i}} I_{2} .
$$

According to Lyapunov theory, system (7) is asymptotically stable if there exists a Lyapunov function $\mathcal{V}_{i}\left(\hat{x}_{[i]}\right)=$ $\hat{x}_{[i]}^{T} P_{i} \hat{x}_{[i]}$, with $P_{i}=P_{i}^{T}>0$, such that

$$
Q_{i}=\left(\hat{A}_{i i}+\hat{B}_{i} K_{i}\right)^{T} P_{i}+P_{i}\left(\hat{A}_{i i}+\hat{B}_{i} K_{i}\right)=F_{i}^{T} P_{i}+P_{i} F_{i}
$$

is negative definite.

In presence of electric interactions between DGUs, the previous conditions are no longer sufficient for asymptotic stability of system (6). In the sequel, we will show that this property is still guaranteed under the following assumptions.

Assumption 1 Each matrix gain $K_{i}, i \in \mathcal{D}$ is designed using $P_{i}>0$ in (9) with the following structure

$$
P_{i}=\left[\begin{array}{c|c}
\eta_{i} I_{2} & \mathbf{0}_{2} \\
\hline \mathbf{0}_{2} & P_{22, i}
\end{array}\right], \quad P_{22, i}=P_{22, i}^{T} \in \mathbb{R}^{4 \times 4} \quad \eta_{i}>0
$$

Assumption 2 Let $\bar{\sigma}>0$ be a constant parameter, common to all DGUs. Parameters $\eta_{i}$ in (10) are given by $\eta_{i}=\bar{\sigma} C_{t i}, \forall i \in \mathcal{D}$.

The next proposition, proved in (Tucci and FerrariTrecate 2018), shows that Assumption 1 implies marginal stability (but not asymptotic stability) of (6).

Proposition 1 Under Assumption 1, the matrix $Q_{i}$ in (9) cannot be negative definite. Moreover,

$$
Q_{i} \leq 0
$$

implies that $Q_{i}$ has the following structure:

$$
Q_{i}=\left[\begin{array}{ccc}
\mathbf{0}_{2} & \mathbf{0}_{2} & \mathbf{0}_{2} \\
\mathbf{0}_{2} & \mathcal{Q}_{22, i} & \mathcal{Q}_{23, i} \\
\mathbf{0}_{2} & \mathcal{Q}_{23, i} & \mathcal{Q}_{33, i}
\end{array}\right]
$$

Next, we consider the overall closed-loop ImG model, given by

$$
\left\{\begin{array}{l}
\dot{\hat{\mathbf{x}}}(t)=(\hat{\mathbf{A}}+\hat{\mathbf{B}} \mathbf{K}) \hat{\mathbf{x}}(t)+\hat{\mathbf{M}} \hat{\mathbf{d}}(t) \\
\hat{\mathbf{y}}(t)=\hat{\mathbf{C}} \hat{\mathbf{x}}(t) \\
\mathbf{z}(t)=\hat{\mathbf{H}} \hat{\mathbf{y}}(t)
\end{array}\right.
$$

where $\mathbf{K}=\operatorname{diag}\left(K_{1}, \ldots, K_{N}\right)$. Being $\mathbf{P}=\operatorname{diag}\left(P_{1}, \ldots, P_{N}\right)$, the collective Lyapunov function is

$$
\mathcal{V}(\hat{\mathbf{x}})=\sum_{i=1}^{N} \mathcal{V}_{i}\left(\hat{x}_{[i]}\right)=\hat{\mathbf{x}}^{T} \mathbf{P} \hat{\mathbf{x}}
$$

and one has that $\dot{\mathcal{V}}(\hat{\mathbf{x}})=\hat{\mathbf{x}}^{T} \mathbf{Q} \hat{\mathbf{x}}$, where

$$
\mathbf{Q}=(\hat{\mathbf{A}}+\hat{\mathbf{B}} \mathbf{K})^{T} \mathbf{P}+\mathbf{P}(\hat{\mathbf{A}}+\hat{\mathbf{B}} \mathbf{K}) .
$$

From Proposition 1, we know that, if Assumption 1 holds, then (i) matrix $\mathbf{Q}$ cannot be negative definite, and (ii), at most, one can have

$$
\mathbf{Q} \leq 0
$$

In the next Proposition, we show that (15) is always satisfied if also Assumption 2 is fulfilled.

Proposition 2 Under Assumptions 1 and 2, if matrix gains $K_{i}$ guarantee (11) for all $i \in \mathcal{D}$, then (15) holds.

PROOF. We start by decomposing the matrix $\hat{\mathbf{A}}$ as follows

$$
\hat{\mathbf{A}}=\hat{\mathbf{A}}_{\mathbf{D}}+\hat{\mathbf{A}}_{\Xi}+\hat{\mathbf{A}}_{\mathbf{C}},
$$

where (i) $\hat{\mathbf{A}}_{\mathbf{D}}=\operatorname{diag}\left(\hat{A}_{i i}, \ldots, \hat{A}_{N N}\right)$ collects the local dynamics only, (ii) $\hat{\mathbf{A}}_{\Xi}=\operatorname{diag}\left(\hat{A}_{\xi 1}, \ldots, \hat{A}_{\xi N}\right)$ with

$$
\hat{A}_{\xi i}=\frac{1}{C_{t i}}\left[\begin{array}{cc|c}
\sum_{j \in \mathcal{N}_{i}}-\widetilde{R}_{i j} & \sum_{j \in \mathcal{N}_{i}}-\widetilde{X}_{i j} & \mathbf{0} \\
\sum_{j \in \mathcal{N}_{i}} \widetilde{X}_{i j} & \sum_{j \in \mathcal{N}_{i}}-\widetilde{R}_{i j} & \mathbf{0} \\
\hline \mathbf{0} & \mathbf{0} & \mathbf{0}
\end{array}\right],
$$

$\widetilde{R}_{i j}=\frac{R_{i j}}{Z_{i j}^{2}}$ and $\widetilde{X}_{i j}=\frac{X_{i j}}{Z_{i j}^{2}}$, takes into account the dependence of each local state on the neighboring DGUs, and (iii) $\hat{\mathbf{A}}_{\mathbf{C}}$ includes the effect of couplings. Notably, $\hat{\mathbf{A}}_{\mathbf{C}}$ is composed by zero blocks on the diagonal and blocks $\hat{A}_{i j}$, $i \neq j$ outside the diagonal.

Using (16), condition (15) becomes

$$
\begin{aligned}
\underbrace{\left(\hat{\mathbf{A}}_{\mathbf{D}}+\hat{\mathbf{B}} \mathbf{K}\right)^{\mathbf{T}} \mathbf{P}+\mathbf{P}\left(\hat{\mathbf{A}}_{\mathbf{D}}\right.}_{(a)}+\hat{\mathbf{B} \mathbf{K})}+\underbrace{\hat{\mathbf{A}}_{\Xi}^{\mathbf{T}} \mathbf{P}+\mathbf{P} \hat{\mathbf{A}}_{\Xi}}_{(b)}+ \\
+\underbrace{\hat{\mathbf{A}}_{\mathbf{C}}^{T} \mathbf{P}+\mathbf{P} \hat{\mathbf{A}}_{\mathbf{C}}}_{(c)} \leq 0 .
\end{aligned}
$$


Since (11) holds, we have that $(a)=\operatorname{diag}\left(Q_{1}, \ldots, Q_{N}\right) \leq$ 0 . Next, we study the matrix $(b)+(c)$ in (18). By construction (recalling (10) and (17)), one can easily show that the matrix $(b)$ is block diagonal, collecting, on its diagonal, blocks $\hat{A}_{\xi i}^{T} P_{i}+P_{i} \hat{A}_{\xi i}$ in the form

$$
\hat{A}_{\xi i}^{T} P_{i}+P_{i} \hat{A}_{\xi i}=\left[\begin{array}{cc|c}
-\sum_{j \in \mathcal{N}_{i}} 2 \tilde{\eta}_{i j} & 0 & \mathbf{0} \\
0 & -\sum_{j \in \mathcal{N}_{i}} 2 \tilde{\eta}_{i j} & \mathbf{0} \\
\hline \mathbf{0} & \mathbf{0} & \mathbf{0}
\end{array}\right]
$$

with $\tilde{\eta}_{i j}=\frac{\eta_{i}}{C_{t i}} \widetilde{R}_{i j}=\bar{\sigma} \widetilde{R}_{i j}$. Regarding matrix (c), we have that each the block in position $(i, j)$ is equal to

$$
\begin{cases}\hat{A}_{j i}^{T} P_{j}+P_{i} \hat{A}_{i j} & \text { if } j \in \mathcal{N}_{i} \\ \mathbf{0} & \text { otherwise }\end{cases}
$$

Using Assumption 2, by direct calculation one obtains

$$
\hat{A}_{j i}^{T} P_{j}+P_{i} \hat{A}_{i j}==\left[\begin{array}{c|c}
2 \tilde{\eta}_{i j} I_{2} & \mathbf{0} \\
\hline \mathbf{0} & \mathbf{0}
\end{array}\right] .
$$

By looking at (19) and (21), we observe that only the elements in position $(1,1)$ and $(2,2)$ of each $6 \times 6$ block of $(b)+(c)$ can be different from zero. Therefore, the positive/negative definiteness of the $6 N \times 6 N$ matrix $(b)+(c)$ can be equivalently studied by considering the $2 N \times 2 N$ matrix

$$
\mathcal{L}=\left[\begin{array}{cccc}
\Phi_{11} & \Phi_{12} & \ldots & \Phi_{1 N} \\
\Phi_{21} & \ddots & \ddots & \vdots \\
\vdots & \ddots & \Phi_{N-1 N-1} & \Phi_{N-1 N} \\
\Phi_{N 1} & \ldots & \Phi_{N N-1} & \Phi_{N N}
\end{array}\right]
$$

obtained by deleting the last four rows and columns in each block of $(b)+(c)$. Furthermore, one has

$$
\Phi_{i i}=\left[\begin{array}{cc}
\sum_{j \in \mathcal{N}_{i}}-2 \tilde{\eta}_{i j} & 0 \\
0 & \sum_{j \in \mathcal{N}_{i}}-2 \tilde{\eta}_{i j}
\end{array}\right], \quad i \in \mathcal{D}
$$

and, using (20) and (21), for $i, j \in \mathcal{D}, i \neq j$, one obtains

$$
\Phi_{i j}= \begin{cases}{\left[\begin{array}{cc}
2 \tilde{\eta}_{i j} & 0 \\
0 & 2 \tilde{\eta}_{i j}
\end{array}\right]} & \text { if } j \in \mathcal{N}_{i} \\
\mathbf{0}_{2} & \text { otherwise }\end{cases}
$$

We notice that $\mathcal{L}$ is symmetric, with non negative offdiagonal elements and zero row and column sum. In other words, $\mathcal{L}$ is a Laplacian matrix, and, as such, it is negative semidefinite. This allows us to show that (18) (and, equivalently, (15)) holds.
Remark 1 As shown in the proof of Proposition 2, Assumption 2 provides a reference setting for showing that the Lyapunov function $\mathcal{V}(\hat{\mathbf{x}})$ cannot increase because of the interactions between DGUs.The assumption is restrictive as it requires perfect knowledge of the capacitances at PCCs. However, thanks to the integral action in local controllers, one expects robustness of stability (Skogestad and Postlethwaite 1996) to sufficiently small perturbations of the DGU parameters.

Asymptotic stability of the ImG, will be discussed in Section 3.2, after providing the algorithm for local control design.

\subsection{Design of local controllers}

For computing local control gains $K_{i}, i \in \mathcal{D}$ guaranteeing (11), we parametrize the unknown quantities in (9) as follows

$$
P_{i}=Y_{i}^{-1}, \quad K_{i}=G_{i} Y_{i}^{-1},
$$

where $G_{i}=\left[\begin{array}{lll}\mathcal{G}_{11, i} & \mathcal{G}_{12, i} & \mathcal{G}_{13, i}\end{array}\right] \in \mathbb{R}^{2 \times 6}, \mathcal{G}_{11, i}, \mathcal{G}_{12, i}, \mathcal{G}_{13, i} \in$ $\mathbb{R}^{2 \times 2}$ and, under Assumption 1 ,

$$
Y_{i}=\left[\begin{array}{c|cc}
\eta_{i}^{-1} I_{2} & \mathbf{0}_{2} & \mathbf{0}_{2} \\
\hline \mathbf{0}_{2} & \mathcal{Y}_{22, i} & \mathcal{Y}_{23, i} \\
\mathbf{0}_{2} & \mathcal{Y}_{23, i}^{T} & \mathcal{Y}_{33, i}
\end{array}\right]=\left[\begin{array}{c|c}
\eta_{i}^{-1} I_{2} & \mathbf{0} \\
\hline \mathbf{0} & Y_{22, i}
\end{array}\right]
$$

We also focus on the matrix

$$
\tilde{Q}_{i}=Y_{i} Q_{i} Y_{i}
$$

instead of $Q_{i}$. Apparently, when $Y_{i}>0, Q_{i}$ is negative semidefinite if and only if $\tilde{Q}_{i}$ has the same property. The advantage of the parametrization (23) is that, as shown below, all entries of $\tilde{Q}_{i}$ depend linearly on $G_{i}$ and $Y_{i}$, while products of matrices $P_{i}$ and $K_{i}$ appear in $Q_{i}$ (see (9)). Indeed, (23) is routinely used for mapping the design of state-feedback gains into LMIs (Boyd et al. 1994).

By direct calculation, from (23), (25), and (9) one has

$$
\begin{aligned}
\tilde{Q}_{i} & =\left[\begin{array}{ccc}
\mathbf{0}_{2} & \tilde{\mathcal{Q}}_{12, i} & \tilde{\mathcal{Q}}_{13, i} \\
\tilde{\mathcal{Q}}_{12, i}^{T} & \tilde{\mathcal{Q}}_{22, i} & \tilde{\mathcal{Q}}_{23, i} \\
\tilde{\mathcal{Q}}_{23, i}^{T} & \tilde{\mathcal{Q}}_{23, i}^{T} & \mathbf{0}_{2}
\end{array}\right] \\
\tilde{\mathcal{Q}}_{12, i} & =\frac{1}{C_{t i}} \mathcal{Y}_{22, i}-\frac{1}{L_{t i} \eta_{i}} I_{2}+\frac{1}{L_{t i}} \mathcal{G}_{11, i}^{T} \\
\tilde{\mathcal{Q}}_{22, i} & =\hat{\mathcal{A}}_{22, i} \mathcal{Y}_{22, i}+\mathcal{Y}_{22, i} \hat{\mathcal{A}}_{22, i}^{T}+\frac{1}{L_{t i}} \mathcal{G}_{12, i}+\frac{1}{L_{t i}} \mathcal{G}_{12, i}^{T} \\
\tilde{\mathcal{Q}}_{13, i} & =\frac{1}{C_{t i}} \mathcal{Y}_{23, i}-\eta_{i}^{-1} I \\
\tilde{\mathcal{Q}}_{23, i} & =\hat{\mathcal{A}}_{22, i} \mathcal{Y}_{23, i}+\frac{1}{L_{t i}} \mathcal{G}_{13, i} .
\end{aligned}
$$

The following result, proved in (Tucci and FerrariTrecate 2018), mirrors Proposition 1 and provides key 
properties of the blocks of $\tilde{Q}_{i}$ that will be used for control design.

\section{Proposition 3 Under Assumption 1,}

(i) the matrix $\tilde{Q}_{i}$ in (26) cannot be negative definite;

(ii) $\tilde{Q}_{i} \leq 0$ implies

$$
\tilde{Q}_{i}=\left[\begin{array}{ccc}
\mathbf{0}_{2} & \mathbf{0}_{2} & \mathbf{0}_{2} \\
\mathbf{0}_{2} & \tilde{\mathcal{Q}}_{22, i} & \mathbf{0}_{2} \\
\mathbf{0}_{2} & \mathbf{0}_{2} & \mathbf{0}_{2}
\end{array}\right],
$$

where $\tilde{\mathcal{Q}}_{22, i}$ is given in (28).

Hereafter, we focus on the design of the local control gains that, using the notation of Section 3.1, can be summarized as follows.

Problem 1 Under Assumptions 1 and 2, compute matrices $K_{i}, i \in \mathcal{D}$ and $P_{22, i}>0$ such that (11) holds.

If $P_{22, i}>0$, then $Y_{22, i}=P_{22 . i}^{-1}>0$. This implies $Y_{i}>0$ and hence $Q_{i} \leq 0 \Leftrightarrow \tilde{Q}_{i} \leq 0$. By means of Proposition 3 -(ii), the last inequality is equivalent to

$$
\begin{aligned}
& \tilde{\mathcal{Q}}_{22, i} \leq 0 \\
& \tilde{\mathcal{Q}}_{13, i}=0, \quad \tilde{\mathcal{Q}}_{23, i}=0, \text { and } \tilde{\mathcal{Q}}_{12, i}=0
\end{aligned}
$$

Therefore, Problem 1 can be rephrased as follows.

Problem 2 Under Assumptions 1 and 2, compute matrices $Y_{22, i}>0$ and $G_{i}, i \in \mathcal{D}$ such that (32) and (33) hold.

We highlight that the inequality (32) can be always verified strictly, as shown in the following proposition, proved in (Tucci and Ferrari-Trecate 2018)

Proposition 4 For any positive-definite matrix $\Gamma_{i} \in$ $\mathbb{R}^{2 \times 2}$ there exist matrices $\mathcal{G}_{12, i}$ and $\mathcal{Y}_{22, i}=\mathcal{Y}_{22, i}^{T}>0$ verifying

$$
\tilde{\mathcal{Q}}_{22, i} \leq-\mathcal{Y}_{22, i} \Gamma_{i}^{-1} \mathcal{Y}_{22, i}
$$

We notice that, from (34), the matrix $\Gamma_{i}^{-1}$ can be interpreted as a robustness margin in the fulfillment of the inequality $\mathcal{Y}_{22, i}^{-1} \tilde{\mathcal{Q}}_{22, i} \mathcal{Y}_{22, i}^{-1} \leq 0$.

Next, we discuss equations (33). From (28), (29), and (27) they are equivalent, respectively, to

$$
\begin{aligned}
\mathcal{Y}_{23, i} & =\frac{C_{t i}}{\eta_{i}} I_{2}, \\
\mathcal{G}_{13, i} & =-\frac{L_{t i} C_{t i}}{\eta_{i}} \hat{\mathcal{A}}_{22, i}, \\
\mathcal{G}_{11, i} & =\frac{L_{t i}}{C_{t i}} \mathcal{Y}_{22, i}+\frac{1}{\eta_{i}} I_{2} .
\end{aligned}
$$

Therefore, Problem 2 can be stated in the following final form.

Problem 3 Under Assumptions 1 and 2, for a given matrix $\Gamma_{i}>0$, find $\mathcal{Y}_{22, i}=\mathcal{Y}_{22, i}^{T}>0, \mathcal{Y}_{33, i}=\mathcal{Y}_{33, i}^{T}>0$ and $\mathcal{G}_{12, i}$ verifying $(34)$ and $Y_{22, i}>0$ (with block $\mathcal{Y}_{23, i}$ in (24) given by (35)).

From Proposition 4, Problem 3 can be always solved if there is $\mathcal{Y}_{33, i}=\mathcal{Y}_{33, i}^{T}>0$ such that $Y_{22, i}>0$. Using the Schur complement (Boyd et al. 1994) on the block structure of $Y_{22, i}$ (shown in (24)), the latter condition is equivalent to $\mathcal{Y}_{33, i}-\mathcal{Y}_{23, i}^{T} \mathcal{Y}_{22, i}^{-1} \mathcal{Y}_{23, i}>0$. From (35) one obtains $\mathcal{Y}_{33, i}-\frac{C_{t i}^{2}}{\eta_{i}^{2}} \mathcal{Y}_{22, i}^{-1}>0$, which is verified by, e.g., $\mathcal{Y}_{33, i}=2 \frac{C_{t i}^{2}}{\eta_{i}^{2}} \mathcal{Y}_{22, i}^{-1}$.

After solving Problem 3, blocks $\mathcal{G}_{13, i}$ and $\mathcal{G}_{11, i}$ of the matrix $G_{i}$ can be computed as in (36) and (37). Furthermore, the controller $K_{i}$ can be recovered from (23). Most importantly, as shown in the next theorem, local controllers guarantee asymptotic stability of the ImG.

Theorem 1 If Assumptions 1 and 2 are fulfilled, local control gains $K_{i} i \in \mathcal{D}$ are computed by solving Problem 3, and the graph $\mathcal{G}_{\text {el }}$ is connected, then system (13) is asymptotically stable.

The proof of Theorem 1, which is based on the Proposition 2 and the Lasalle's invariance theorem, can be found in (Tucci and Ferrari-Trecate 2018).

\subsection{Optimization-based design of local controllers}

It is not difficult to see that a solution to Problem 3 is provided by the following LMI optimization problem

$$
\begin{aligned}
& \mathcal{O}_{i}: \min _{\mathcal{Y}_{22, i}, \mathcal{Y}_{33, i}, \mathcal{G}_{12, i},} \alpha_{1 i} \gamma_{1 i}+\alpha_{2 i} \gamma_{2 i}+\alpha_{3 i} \beta_{i}+\alpha_{4 i} \zeta_{i} \\
& Y_{i}=\left[\begin{array}{ccc}
\eta_{i}^{-1} I_{2} & \mathbf{0}_{2} & \mathbf{0}_{2} \\
\mathbf{0}_{2} & \mathcal{Y}_{22, i} & \frac{C_{t i}}{\eta_{i}} I_{2} \\
\mathbf{0}_{2} & \frac{C_{t i}}{\eta_{i}} I_{2} & \mathcal{Y}_{33, i}
\end{array}\right]>0 \\
& {\left[\begin{array}{rr}
\hat{\mathcal{A}}_{22, i} \mathcal{Y}_{22, i}+\mathcal{Y}_{22, i} \hat{\mathcal{A}}_{22, i}^{T}+\frac{1}{L_{t i}} \mathcal{G}_{12, i}+\frac{1}{L_{t i}} \mathcal{G}_{12, i}^{T} & \mathcal{Y}_{22, i} \\
\mathcal{Y}_{22, i} & -\Gamma_{i}
\end{array}\right] \leq 0} \\
& {\left[\begin{array}{cc}
-\beta_{i} I & G_{i}^{T} \\
G_{i} & -I
\end{array}\right]<0, \quad\left[\begin{array}{cc}
Y_{i} & I \\
I & \zeta_{i} I
\end{array}\right]>0} \\
& \gamma_{1 i}>0, \quad \gamma_{2 i}>0, \quad \beta_{i}>0, \quad \zeta_{i}>0
\end{aligned}
$$

where $\alpha_{k i}, k=1, \ldots, 4$ are positive weights and $\Gamma_{i}=$ $\operatorname{diag}\left(\gamma_{1 i}, \gamma_{2 i}\right)$. Indeed, (38b) corresponds, up to Schur complement, to (34). Furthermore, constraints (38c) are always feasible. Their role is to penalize aggressive control actions (Riverso et al. 2015) since they correspond to the bound $\left\|K_{i}\right\|_{2} \leq \sqrt{\beta_{i}} \zeta_{i}$ and large values of $\beta_{i}$ and $\zeta_{i}$ 
are are penalized in the cost function. Finally, the minimization of $\gamma_{1 i}$ and $\gamma_{2 i}$ corresponds to the maximization of the robustness margin $\Gamma_{i}^{-1}$ discussed in the previous section.

We highlight that the computation of controller $\mathcal{C}_{[i]}$ is decentralized (i.e. independent of the synthesis of controllers $\mathcal{C}_{[j]}, j \neq i$ ), up to the knowledge, shared by all DGUs, of the parameter $\bar{\sigma}$ in Assumption 2. Indeed, constraints in (38) depend only upon local electrical parameters of DGU $i$ and local design parameters $\left(\alpha_{k i}\right.$, $k=1, \ldots, 4)$.

Remark 2 As shown in (Riverso et al. 2014), if a DGU is disconnected from the ImG, its closed-loop performance can be finely tuned by complementing local controller with pre-filters and load-current compensators. These enhancements, which are standard in linear control systems, are usually effective also when DGUs are interconnected. However, a rigorous assessment of performance cannot be based only on local line-independent closed-loop DGU models. Indeed, even if tracking is perfect (i.e. DGUs are ideal voltage sources) the ImG dynamics depends on the parameters of $R L$ lines and the Im $G$ topology. Moreover, even if QSL approximations hold and the ImG is asymptotically stable, the Lyapunov function (14) does not capture the exponential convergence rate of the $\operatorname{Im} G$ to the equilibrium state because $\mathbf{Q}$ is not negative definite.

\subsection{PnP operations}

Suppose that we want to add the DGU $\left.\hat{\Sigma}_{[N+1]}^{D G U}\right)$ to the ImG. From Theorem 1, in order to preserve stability, it is enough to equip $\hat{\Sigma}_{[N+1]}^{D G U}$ with the controller obtained by solving the LMI problem $\mathcal{O}_{N+1}$ and connect the DGU to the ImG. Differently from (Riverso et al. 2015), the plug-in of a DGU is never denied and no other DGU must to retune its local controller.

The unplugging of a DGU is even simpler as it has no impact on the controllers of the remaining units. Therefore, if $\mathcal{G}_{e l}$ remains connected, the stability of the ImG is preserved. Otherwise, if $\mathcal{G}_{e l}$ becomes disconnected, Theorem 1 can still be applied to each connected component of $\mathcal{G}_{e l}$ (which, in turn, can be seen as an independent ImG). An example is provided in Section 4.

\section{Simulation results}

For studying performance brought about by $\mathrm{PnP}$ controllers, we consider the 10-DGU, meshed ImG in Figure 2 . In order to simulate a realistic scenario, we replace constant current loads with RL loads, except for DGU 2 which is connected to a three-phase six-pulse diode rectifier. The values of electrical parameters are similar to those provided in (Riverso et al. 2015).

The simulation (conducted in PSCAD) starts with DGUs 1-9 connected together and equipped with PnP controllers $\mathcal{C}_{[i]}, i=1, \ldots, 9$.

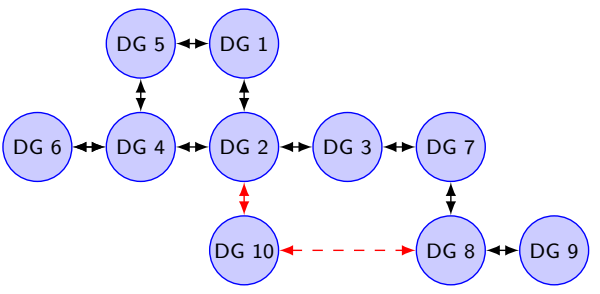

(a) ImG topologies until $t=12 \mathrm{~s}$. For $0 \leq t<7.5 \mathrm{~s}$, DGUs 1-9 are interconnected (in black); at $t=7.5$ $\mathrm{s}$, DGU 10 joins the network (in red).
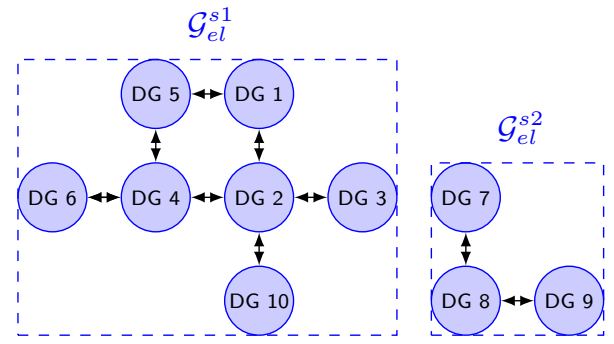

(b) Independent ImGs after the trip of lines 3-7 and $8-10$ at time $t=12 \mathrm{~s}$.

Fig. 2. Simulation of a 10-DGUs ImGs: considered network topologies.

At time $t=7.5 \mathrm{~s}$, we connect DGU 10 with DGUs 2 and 8 (see Figure $2 \mathrm{a}$ ). The $d q$ component of the voltages at PCCs 2, 8, 10 are shown in Figures 3a, 3d and 3e, respectively. Notably, we notice very small deviations of the DGUs voltages from their reference signals $\left(V_{2, \text { ref }}^{d}=0.6 \mathrm{pu}, V_{2, \text { ref }}^{q}=0.5 \mathrm{pu}, V_{8, \text { ref }}^{d}=0.7 \mathrm{pu}\right.$, $V_{8, \text { ref }}^{q}=0.6 \mathrm{pu}$, and $\left.V_{10, \text { ref }}^{q}=0.8 \mathrm{pu}, V_{10, \text { ref }}^{q}=0.6 \mathrm{pu}\right)$. The oscillations of $V_{2}^{d}$ and $V_{2}^{q}$ around their respective references are due to the fact that the corresponding signals in the $a b c$ reference frame are not perfectly sinusoidal because of the nonlinear behavior of the rectifier. For analyzing the robustness of the PnP-controlled ImG against load dynamics, at time $t=10 \mathrm{~s}$ the $R L$ load at PCC 10 changes from $R=60 \Omega, L=0.02 \mathrm{mH}$ to $R=120 \Omega, L=0.02 \mathrm{mH}$. From Figures 3a, 3d and 3e, we notice that the $d$ and $q$ components of the voltages at PCCs 2, 8 and 10, do not significantly deviate from their references. Figure $3 \mathrm{f}$ shows that the real-time plugin of DGU 10 and the load change at its PCC produce minor effects also on the frequency profiles of the PCC voltages (veriations around the $50 \mathrm{~Hz}$ reference value are smaller than $0.6 \mathrm{~Hz}$ ). In a similar way, there are no significant deviations from the reference RMS voltages (see Figure 3g).

For testing the capabilities of $\mathrm{PnP}$ regulators to preserve stability after line faults, at time $t=12 \mathrm{~s}$, we simulate the simultaneous trip of lines 3-7 and 8-10, leading to the formation of two independent ImGs (see the subgraphs $\mathcal{G}_{e l}^{s 1}$ and $\mathcal{G}_{e l}^{s 2}$ Figure 2b). As described in Section 3.4 , the stability of the two networks is preserved (see Figures 3b-3g), without the need to redesign any local 
controller. Finally, from Figure 3 h, we notice that the Total Harmonic Distortion (THD) of the voltage at PCC 2 (whose local load is nonlinear) always remains below $5 \%$, which is the maximum limit recommended by IEEE standards.

\subsection{Impact of clock desynchronization}

As mentioned in the introduction, we assume the ImG is equipped with a clock synchronization layer capable to guarantee a worst-case accuracy in presence of clock drifts. In the following, we discuss the ImG performance in the pessimistic scenario where clock shifts are constant and maximal. A phase shift in the rotating $d q$ frame generates an offset in the $d q$ signals, i.e. an additive step disturbance on the $d q$ side of the $a b c \rightarrow d q$ and $d q \rightarrow a b c$ transformation blocks in Figure 1. As an example, assuming $\omega_{0}=2 \pi 50[\mathrm{rad} / \mathrm{s}]$ and using the protocols in (IEEE1588 2008, IEEE1588 2017), which provide a clock accuracy of $10^{-6} \mathrm{~s}$, one obtains a worst-case phase shift $\tilde{\theta}_{0, \mathrm{DGU}_{i}}=e_{\mathrm{DGU}_{i}} \cdot \omega_{0} \cdot \frac{180}{\pi}=\left(3.6 \times 10^{-2}\right)^{\circ}$. On the one hand, step disturbances on the feedback path cannot be rejected by the controller (which contains an integral action) and induce a tracking offset. On the other hand, since the closed-loop system is linear, asymptotic stability of the ImG is not compromised by exogenous disturbances. For simulations under significant clock shifts (i.e. much higher than those reported in (IEEE1588 2008, IEEE1588 2017)), we defer the reader to (Tucci and Ferrari-Trecate 2018).

\section{Conclusions}

In this paper, we presented a decentralized control approach to voltage and frequency stabilization in $\mathrm{AC}$ ImG. The design algorithm is always feasible and guarantees overall ImG stability while computing local controllers in a line-independent fashion. In future research we will study how to include performance specifications in local control synthesis. Another interesting research topic is how to extend $\mathrm{PnP}$ design to microgrids including both synchronous and inverter-interfaced distributed generators.

\section{References}

Babazadeh, M. and H. Karimi (2013). A Robust Two-Degree-ofFreedom Control Strategy for an Islanded Microgrid. IEEE Transactions on Power Delivery 28(3), 1339-1347.

Boyd, S., L. El Ghaoui, E. Feron and V. Balakrishnan (1994). Linear matrix inequalities in system and control theory. SIAM Studies in Applied Mathematics, vol. 15. Philadelphia, Pennsylvania, USA.

Dörfler, F. and F. Bullo (2013). Kron reduction of graphs with applications to electrical networks. IEEE Transactions on Circuits and Systems I: Regular Papers 60(1), 150-163.
Dörfler, F., J. W. Simpson-Porco and F. Bullo (2014). Plug-andplay control and optimization in microgrids. In: 53rd IEEE Conference on Decision and Control. pp. 211-216.

Etemadi, A. H., E. J. Davison and R. Iravani (2012). A Decentralized Robust Control Strategy for MultiDER Microgrids - Part I: Fundamental Concepts. IEEE Transactions on Power Delivery 27(4), 1843-1853.

Floriduz, A., M. Tucci, S. Riverso and G. Ferrari-Trecate (2018). Approximate kron reduction methods for electrical networks with applications to plug-and-play control of AC islanded microgrids. IEEE Transactions on Control Systems Technology pp. 1-14.

Guerrero, J. M., M. Chandorkar, T. Lee and P. C. Loh (2013). Advanced Control Architectures for Intelligent Microgrids - Part I: Decentralized and Hierarchical Control. IEEE Transactions on Industrial Electronics 60(4), 1254-1262.

IEEE1588 (2008). IEEE standard for a precision clock synchronization protocol for networked measurement and control systems. IEEE Std 1588-2008 (Revision of IEEE Std 1588-2002) pp. 1-300.

IEEE1588 (2017). IEEE standard profile for use of IEEE 1588 precision time protocol in power system applications. IEEE C37.238-2017, August 2017 pp. 1-42.

Kolluri, R.R., I. Mareels, Alpcan T., M. Brazil, J. de Hoog and D.A. Thomas (2018). Stability and active power sharing in droop controlled inverter interfaced microgrids: Effect of clock mismatches. Automatica 93, 469 - 475.

Riverso, S., F. Sarzo and G. Ferrari-Trecate (2014). Plugand-play voltage and frequency control of islanded microgrids with meshed topology. Technical report. https://arxiv.org/abs/1405.2421.

Riverso, S., F. Sarzo and G. Ferrari-Trecate (2015). Plug-andplay voltage and frequency control of islanded microgrids with meshed topology. IEEE Transactions on Smart Grid 6(3), 1176-1184.

Riverso, S., M Tucci, J.C. Vasquez, J.M. Guerrero and G. FerrariTrecate (2018). Stabilizing plug-and-play regulators and secondary coordinated control for AC islanded microgrids with bus-connected topology. Applied Energy 210, 914-924.

Sadabadi, M. S., Q. Shafiee and A. Karimi (2017). Plug-andplay voltage stabilization in inverter-interfaced microgrids via a robust control strategy. IEEE Transactions on Control Systems Technology 25(3), 781-791.

Schiffer, J., D. Zonetti, R. Ortega, A. M Stanković, T. Sezi and J. Raisch (2016). A survey on modeling of microgrids From fundamental physics to phasors and voltage sources. Automatica 74, 135-150.

Schiffer, J., R. Ortega, A. Astolfi, J. Raisch and T. Sezi (2014). Conditions for stability of droop-controlled inverter-based microgrids. Automatica 50(10), 2457-2469.

Simpson-Porco, J. W., F. Dörfler and F. Bullo (2013). Synchronization and power sharing for droop-controlled inverters in islanded microgrids. Automatica 49(9), 26032611.

Skogestad, S. and I. Postlethwaite (1996). Multivariable feedback control: analysis and design. John Wiley \& Sons. New York, NY, USA.

Tucci, M. and G. Ferrari-Trecate (2017). Voltage and frequency control in $\mathrm{AC}$ islanded microgrids: a scalable, line-independent design algorithm. IFAC-PapersOnLine 50(1), 13922 - 13927. 20th IFAC World Congress.

Tucci, M. and G. Ferrari-Trecate (2018). A scalable lineindependent design algorithm for voltage and frequency 


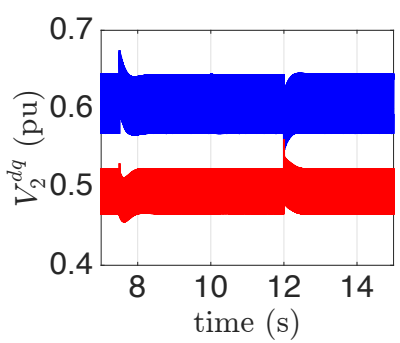

(a) $d q$ components of the voltage at $\mathrm{PCC}_{2}$

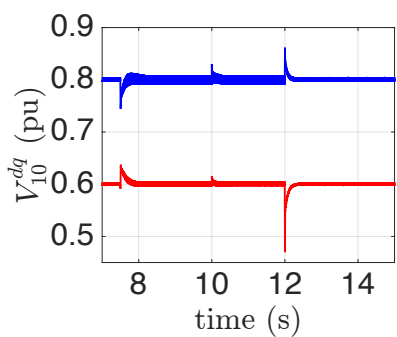

(e) $d q$ components of the voltage at $P C C_{10}$.

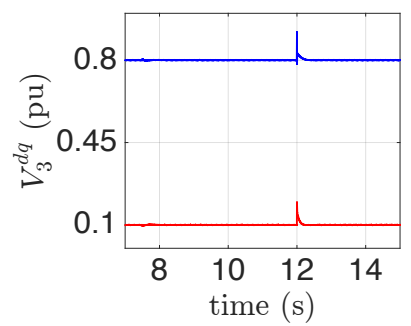

(b) $d q$ components of the voltage at $\mathrm{PCC}_{3}$.

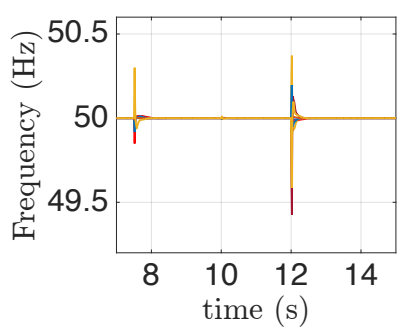

(f) Frequency of phase $a$ at the $P C C$ s of all DGUs.

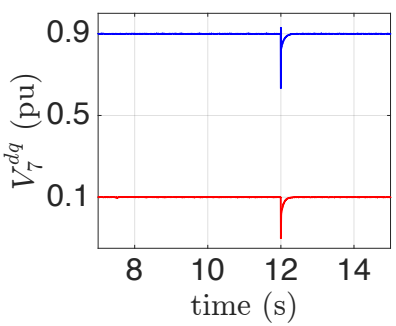

(c) $d q$ components of the voltage at $\mathrm{PCC}_{7}$.

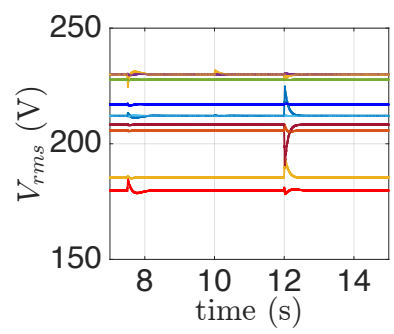

(g) RMS voltages of phase $a$ at the $P C C$ s of all DGUs.

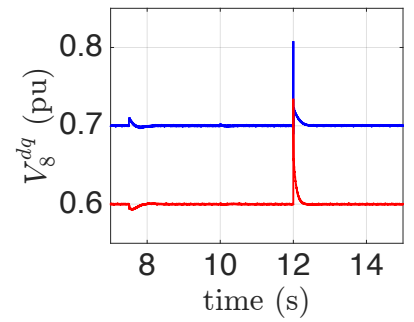

(d) $d q$ components of the voltage at $\mathrm{PCC}_{8}$.

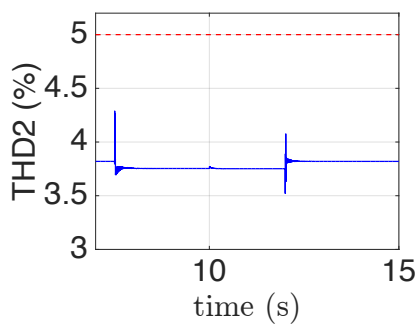

(h) THD of phase $a$ of the voltage at $\mathrm{PCC}_{2}$.

Fig. 3. Performance of PnP voltage and frequency control. Connection of DGU 10, load change at PCC 10, and disconnection of DGUs 3 and 7, are performed at times $t=7.5 \mathrm{~s}, t=10 \mathrm{~s}$ and $t=12 \mathrm{~s}$, respectively. In panels (a)-(e), blue and red lines represent the $d$ and $q$ components, respectively, of voltages.

control in AC islanded microgrids. Technical report. https://arxiv.org/abs/1703.02336.

Tucci, M., S. Riverso and G. Ferrari-Trecate (2018). Line-independent plug-and-play controllers for voltage stabilization in DC microgrids. IEEE Transactions on Control Systems Technology 26(3), 1115-1123. 